\title{
Mechanism of exciton dephasing in a single carbon nanotube studied by photoluminescence spectroscopy
}

\section{$\operatorname{AUTHOR(S):~}$}

Yoshikawa, Kohei; Matsunaga, Ryusuke; Matsuda, Kazunari; Kanemitsu, Yoshihiko

\section{CITATION:}

Yoshikawa, Kohei ... [et al]. Mechanism of exciton dephasing in a single carbon nanotube studied by photoluminescence spectroscopy. Applied Physics Letters 2009, 94(9): 093109.

\section{ISSUE DATE:}

2009-03

URL:

http://hdl.handle.net/2433/87344

RIGHT:

c 2009 American Institute of Physics. 


\title{
Mechanism of exciton dephasing in a single carbon nanotube studied by photoluminescence spectroscopy
}

\author{
Kohei Yoshikawa, ${ }^{1}$ Ryusuke Matsunaga, ${ }^{1}$ Kazunari Matsuda, ${ }^{1, a)}$ and \\ Yoshihiko Kanemitsu, ${ }^{1, \text { b) }}$ \\ ${ }^{1}$ Institute for Chemical Research, Kyoto University, Uji, Kyoto 611-0011, Japan \\ ${ }^{2}$ Photonics and Electronics Science and Engineering Center, Kyoto University, Kyoto 615-8510, Japan
}

(Received 20 November 2008; accepted 7 February 2009; published online 5 March 2009)

\begin{abstract}
We studied the temperature and chirality dependence of the photoluminescence (PL) linewidth of single carbon nanotubes to clarify the mechanism of exciton dephasing. The PL linewidth of a single carbon nanotube broadened linearly with increasing temperature, indicating that the linewidth and exciton dephasing are determined through exciton-phonon interactions. From the chirality dependence of the PL linewidth, we concluded that exciton dephasing is caused by both the longitudinal acoustic and twisting phonon modes. () 2009 American Institute of Physics.
\end{abstract}

[DOI: $10.1063 / 1.3089843]$

The dynamics of electrons and excitons are strongly influenced by phonons. In bulk materials, the loss of coherence (i.e., dephasing) of electrons and excitons occurs by phonon scattering on subpicosecond time scales. ${ }^{1}$ The dephasing time and mechanism depend strongly on the dimensionality and size of the electronic states in low-dimensional semiconductors, such as one-dimensional (1D) quantum wires and zero-dimensional quantum dots. ${ }^{2,3} \mathrm{~A}$ single-walled carbon nanotube (SWNT) is a prototypical 1D electronic system. The optical properties of SWNTs have attracted a great deal of attention, both from the perspective of their fundamental physics, and for their optoelectronic device applications. Potential applications include electroluminescence, ${ }^{4}$ saturable absorbers for ultrafast lasers, ${ }^{5}$ and fluorescent biolabeling. ${ }^{6}$ These optical properties are due to the creation of stable excitons by enhanced Coulomb interactions. ${ }^{7-9}$ The exciton dynamics are dominated by the exciton dephasing and energy relaxation processes, in which the exciton-phonon interactions play an important role. ${ }^{10}$ However, the exciton dephasing mechanism in SWNTs is still poorly understood.

Single carbon nanotube photoluminescence (PL) provides inherent properties of SWNT beyond the ensembleaveraged PL measurements. ${ }^{11-18}$ As the exciton dephasing induced by phonon scattering broadens the linewidths of optical transitions, information on the exciton dephasing mechanism can be obtained directly from the intrinsic spectral linewidth of a single carbon nanotube, free from the inhomogeneous broadening. In this paper, we studied the exciton dephasing using single carbon nanotube PL spectroscopy. The PL linewidth of a single carbon nanotube broadened linearly with temperature, indicating that the linewidth and exciton dephasing were determined by exciton-phonon interactions. From the diameter and chirality dependence of the linewidth, we also investigated the mechanism of the exciton dephasing in detail.

Isolated and suspended SWNTs between the grooves were used as samples. The SWNTs were synthesized on patterned $\mathrm{SiO}_{2}$ and $\mathrm{Si}$ substrates by the alcohol catalytic chemical vapor deposition method. ${ }^{19}$ The substrates were patterned

\footnotetext{
${ }^{a)}$ Electronic mail: matsuda@ @scl.kyoto-u.ac.jp.

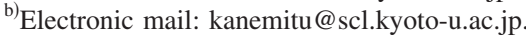

with parallel grooves of typical width and depth of $0.5-5$ and 0.5-5 $\mu \mathrm{m}$, respectively. Several SWNT samples were prepared with different growth temperatures and times, and single nanotube PL spectroscopy was performed on samples grown at $650-800{ }^{\circ} \mathrm{C}$ for $10 \mathrm{~min}$. The average density of luminescent SWNTs in the samples was $0.1-1 / \mu \mathrm{m}^{2}$.

Single SWNT PL measurements were performed using a custom-built variable temperature confocal microscope. The SWNT samples were mounted on a stage and excited with a $\mathrm{He}-\mathrm{Ne}$ laser $(1.959 \mathrm{eV})$ which was focused on the sample surface with a microscope objective (numerical aperture 0.5). The PL signal from the SWNTs was spectrally dispersed by a $30 \mathrm{~cm}$ spectrometer equipped with a liquid-nitrogen $\left(\mathrm{LN}_{2}\right)$-cooled InGaAs photodiode array (spectral range of $0.78-1.38 \mathrm{eV}$ ). The spectral resolution of the system was about $1.0 \mathrm{meV}$. The typical detector accumulation time was $30 \mathrm{~s}$.

Figure 1(a) shows the temperature dependence of the PL spectra of a single SWNT from 40 to $297 \mathrm{~K}$. The SWNT has a chiral index of $(9,8)$, based on the emission energies re-

(a)

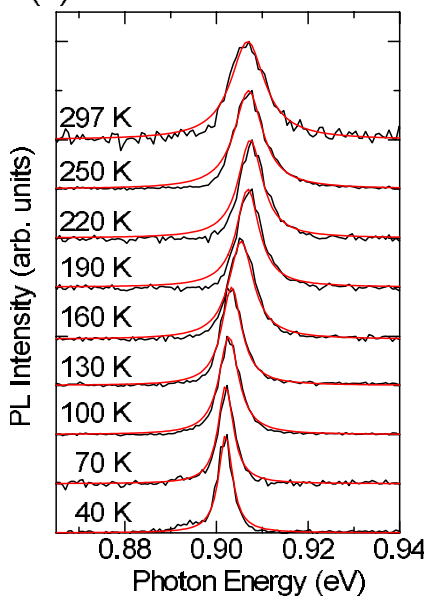

FIG. 1. (Color online) (a) Temperature dependence of the PL spectra of a single SWNT from 40 to $297 \mathrm{~K}$ excited with a $\mathrm{He}-\mathrm{Ne}$ laser light. The smooth lines show fits to the Lorentzian function. (b) Spectral linewidths (FWHM) as a function of temperature. The solid line shows the linear temperature dependence. 
ported in Refs. 20 and 21. We confirmed that the PL spectra come from a single carbon nanotube by PL images (not shown here). ${ }^{14,15}$ The PL spectral shape can be approximately fitted by a single Lorentzian function. The linewidths (full width at half maximum) (FWHM) of the Lorentzian functions are almost dominated by the exciton dephasing time due to exciton-phonon interactions because the exciton lifetime is longer than $30 \mathrm{ps}$, and the contribution to the linewidth from the exciton lifetime is negligibly small. ${ }^{22}$ From the homogeneous linewidth, we calculated the exciton dephasing time at room temperature at about $120 \mathrm{fs}$ for $(9,8)$ nanotubes. The order of the experimentally obtained dephasing time is almost consistent with the ab initio calculation. ${ }^{10}$

The PL spectra become narrower when the temperature decreases, as shown in Fig. 1(a). The linewidth of a single SWNT corresponding to a homogeneous linewidth is plotted as a function of temperature in Fig. 1(b). The temperature dependence of the homogeneous linewidth is usually described as ${ }^{23,24}$

$$
\Gamma=\Gamma_{0}+A T+\frac{B}{\exp \left(\hbar \omega / k_{B} T\right)-1},
$$

where $\Gamma_{0}$ is the residual linewidth at $T=0 \mathrm{~K}$ including the instrumental spectral resolution, and $\hbar \omega$ is the energy of the high frequency phonon modes in SWNTs, such as the radial breathing mode (RBM) and averaged phonon mode discussed in Ref. 25. The coefficients $A$ and $B$ are excitonphonon coupling constants for low- and high-frequency modes, respectively. The solid line in Fig. 1(b) shows the linewidth fitted using Eq. (1). The temperature dependence of the homogeneous linewidth shows an almost linear behavior over a wide temperature range. The value of $A$ obtained for the $(9,8)$ nanotube is $0.020 \mathrm{meV} / \mathrm{K}$. The contribution of the high-frequency modes to the linewidth broadening is much smaller than that of the low-frequency acoustic phonon mode and is negligibly small.

We obtained the PL spectra at $300 \mathrm{~K}$ from more than 200 different isolated SWNTs with a variety of chiral indices. The chiral index assignment for each PL spectrum is based on the emission energy data. ${ }^{20,21}$ The homogeneous linewidth as a function of the nanotube diameter $d$ from the PL spectra of single carbon nanotubes (solid circles) is shown in Fig. 2(a). The smallest values are plotted as the intrinsic linewidth for nanotubes with the same chiral index, because the extrinsic factors (defects, impurities, and so on) cause broadening to the residual linewidth $\Gamma_{0}$. The PL linewidth at $300 \mathrm{~K}$ clearly increases broader as the diameter decreases.

The exciton-phonon coupling constant $A$ for low energy phonon modes is plotted in Fig. 2(b). The diameter dependence of $A$ is well consistent with that of the homogeneous linewidth at $300 \mathrm{~K}$ in Fig. 2(a). These results indicate that the diameter dependence of the PL linewidth observed at $300 \mathrm{~K}$ is not due to the residual linewidth $\Gamma_{0}$ and the nonlinear temperature term in Eq. (1), which is caused by highfrequency modes such as the RBM and averaged phonon mode [see Fig. 1(b)]. From the diameter dependence of the homogeneous linewidth and the exciton-phonon coupling constant $A$, it can be seen that the exciton-phonon interaction is stronger and the exciton dephasing time shorter in tubes of smaller diameter.

Figure 3 shows the chirality dependence of the homogeneous linewidth at $300 \mathrm{~K}$ and the exciton-phonon coupling

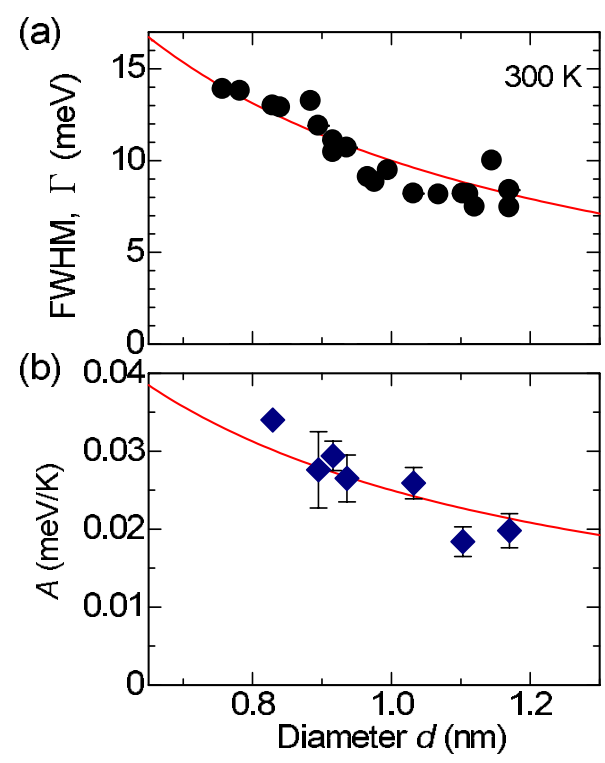

FIG. 2. (Color online) (a) Diameter dependence of the homogeneous linewidth of PL spectra for isolated single SWNTs at $300 \mathrm{~K}$. The solid line corresponds to the result calculated using Eqs. (1) and (2). (b) Diameter dependence of the exciton-phonon coupling constant. The solid line corresponds to the result calculated using Eq. (2).

constant $A$. The data are plotted for SWNTs of around 1.0 $\mathrm{nm}$ in diameter (within the range of $0.9-1.2 \mathrm{~nm}$ ). Both the homogeneous linewidth and the exciton-phonon coupling constant are almost constant, and neither show the clear chiral angle $\theta$ dependence. However, there is the scattering of the data due to the diameter dependence, as discussed below.

We will discuss the mechanism of the exciton dephasing from the diameter and chirality dependence of the excitonphonon coupling (homogeneous linewidth) in detail. Here we refer the discussion on the electron-phonon interaction, because there have been no detailed quantitative theoretical studies of exciton-phonon interactions. As described in Ref. 26 , the diameter and chirality dependence of the excitonphonon interactions show a similar tendency to the electronphonon interactions. The exciton (electron)-phonon coupling constant using the reduced exciton (electron)-phonon matrix element may be written as follows: ${ }^{27,28}$

$$
A \propto \frac{\sqrt{M_{0}}}{d} \cdot \sum_{j}\left|g_{j}\right|^{2} E^{-1 / 2},
$$

where $M_{0}$ is the exciton effective mass, $E$ is the exciton energy, and $g_{j}$ is the reduced matrix-element of the exciton-

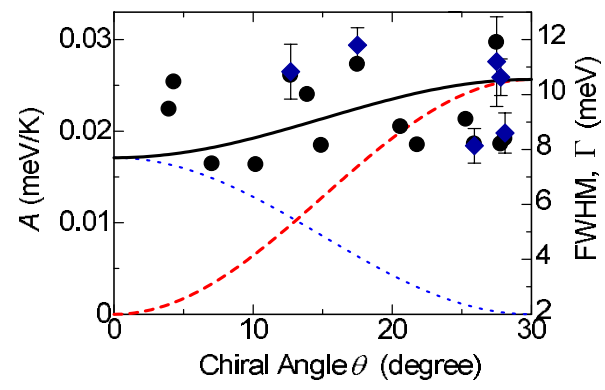

FIG. 3. (Color online) Chirality dependence of the homogeneous linewidth at $300 \mathrm{~K}$ (solid circles) and the exciton-phonon coupling constant (solid diamonds). The solid line corresponds to the calculated chirality dependence. The dotted and dashed lines show the contributions of the TW and LA phonon modes, respectively. 
phonon interaction. As both $M_{0}$ and $E$ are inversely proportional to the diameter $d$, the exciton-phonon coupling in Eq. (2) has $1 / d$ dependence. The diameter dependence $(A$ $\propto 1 / d)$ arises from the specific characteristics of the excitonphonon interaction in 1D carbon nanotubes.

The linear temperature dependence of the linewidth noted above suggests that the phonon modes that contribute to the linewidth broadening are low-energy acoustic modes, such as twisting (TW) and longitudinal acoustic (LA). The chirality dependences of the predicted reduced exciton (electron)-phonon matrix elements for TW and LA phonon modes are described by $g_{\mathrm{TW}} \propto \cos 3 \theta$ and $g_{\mathrm{LA}} \propto 0.81 \sin 3 \theta$, respectively. ${ }^{28}$ Using the reduced matrix-elements, we calculated the diameter dependence of the exciton-phonon coupling constant from Eq. (2). The results are indicated by the solid line in Fig. 2(b). The calculated diameter dependence of the homogeneous linewidth from Eqs. (1) and (2) is also shown in Fig. 2(a) (solid line). We assumed that the residual linewidths are constant $(2.0 \mathrm{meV})$ in the theoretically calculated curve, because they are almost constantly independent of the diameter, and the variation of the values is small (within $\sim 1 \mathrm{meV}$ ) for each single nanotube we observed. Both theoretical calculated lines in Figs. 2(a) and 2(b) agrees closely with the experimental results.

Figure 3 also shows the calculated chirality dependence of the exciton-phonon coupling constant (homogeneous linewidth) for SWNTs of around $1 \mathrm{~nm}$ in diameter, indicated by the solid line. The contributions of the two phonon modes LA and TW as dashed and dotted lines, respectively. The calculated result indicated by the solid line is in agreement with the experimental results of the chirality dependence of the exciton-phonon coupling constant (homogeneous linewidth). In the small (large) chiral angle nanotubes, the TW (LA) mainly contributes to the exciton-phonon coupling constant (homogeneous linewidth). These observations indicate that both the LA and TA modes contribute to the exciton dephasing for carbon nanotubes.

In conclusion, we studied the mechanism of exciton dephasing in SWNTs using single carbon nanotube PL spectroscopy. The PL linewidth of a single carbon nanotube broadened linearly with temperature, indicating that the linewidth was determined by the exciton-acoustic phonon dephasing process. The experimental and theoretical calculations of the chirality dependence of the homogeneous linewidths indicated that the exciton dephasing is caused by both the LA and TW phonon modes

The authors would like to thank Dr. S. Kasai and Professor T. Ono for fabricating the patterned Si substrates. This work was supported in part by a Grant-in-Aid for Scientific Research from JSPS (Japan Society for the Promotion of
Science, Grant No. 20340075) and MEXT (Nos. 20048004 and 20104006), and the CASIO Science Promotion Foundation and Kyoto University G-COE program from MEXT.

${ }^{1}$ P. C. Becker, H. L. Fragnito, C. H. Brito Cruz, R. L. Fork, J. E. Cunningham, J. E. Henry, and C. V. Shank, Phys. Rev. Lett. 61, 1647 (1988).

${ }^{2}$ D. Gammon, E. S. Snow, B. V. Shanabrook, D. S. Katzer, and D. Park, Science 273, 87 (1996)

${ }^{3}$ W. Braun, M. Bayer, A. Forchel, H. Zull, J. P. Reithmaier, A. I. Filin, and T. L. Reinecke, Phys. Rev. B 56, 12096 (1997).

${ }^{4}$ J. A. Misewich, R. Martel, Ph. Avouris, J. C. Tsang, S. Heinze, and J. Tersoff, Science 300, 783 (2003).

${ }^{5}$ A. G. Rozhin, Y. Sakakibara, S. Namiki, M. Tokumoto, H. Kataura, and Y. Achiba, Appl. Phys. Lett. 88, 051118 (2006).

${ }^{6}$ T. K. Leeuw, R. M. Reith, R. A. Simonette, M. E. Harden, P. Cherukuri, D. A. Tsyboulski, K. M. Beckingham, and R. B. Weisman, Nano Lett. 7, 2650 (2007).

${ }^{7}$ T. Ando, J. Phys. Soc. Jpn. 66, 1066 (1997).

${ }^{8}$ C. L. Kane and E. J. Mele, Phys. Rev. Lett. 90, 207401 (2003).

${ }^{9}$ F. Wang, G. Dukovic, L. E. Brus, and T. F. Heinz, Science 308, 838 (2005).

${ }^{10}$ B. F. Habenicht, H. Kamisaka, K. Yamashita, and O. V. Prezhdo, Nano Lett. 7, 3260 (2007).

${ }^{11}$ A. Hartschuh, H. N. Pedrosa, L. Novotny, and T. D. Krauss, Science 301, 1354 (2003)

${ }^{12}$ H. Htoon, M. J. O'Connell, P. J. Cox, S. K. Doorn, and V. I. Klimov, Phys. Rev. Lett. 93, 027401 (2004).

${ }^{13}$ J. Lefebvre, P. Finnie, and Y. Homma, Phys. Rev. B 70, 045419 (2004).

${ }^{14}$ K. Matsuda, Y. Kanemitsu, K. Irie, T. Saiki, T. Someya, Y. Miyauchi, and S. Maruyama, Appl. Phys. Lett. 86, 123116 (2005).

${ }^{15}$ T. Inoue, K. Matsuda, Y. Murakami, S. Maruyama, and Y. Kanemitsu, Phys. Rev. B 73, 233401 (2006).

${ }^{16}$ K. Matsuda, T. Inoue, Y. Murakami, S. Maruyama, and Y. Kanemitsu, Phys. Rev. B 77, 193405 (2008).

${ }^{17}$ R. Matsunaga, K. Matsuda, and Y. Kanemitsu, Phys. Rev. Lett. 101, 147404 (2008).

${ }^{18}$ T. Gokus, A. Hartschuh, H. Harutyunyan, M. Allegrini, F. Hennrich, M. Kappes, A. A. Green, M. C. Hersam, P. T. Araújo, and A. Jorio, Appl. Phys. Lett. 92, 153116 (2008).

${ }^{19}$ S. Maruyama, R. Kojima, Y. Miyauchi, S. Chiashi, and M. Kohno, Chem. Phys. Lett. 360, 229 (2002).

${ }^{20}$ M. J. O'Connell, S. M. Bachilo, X. B. Huffman, V. C. Moore, M. S. Strano, E. H. Haroz, K. L. Rialon, P. J. Boul, W. H. Noon, C. Kittrell, J. Ma, R. H. Hauge, R. B. Weisman, and R. E. Smalley, Science 297, 593 (2002).

${ }^{21}$ J. Lefebvre, J. M. Fraser, Y. Homma, and P. Finnie, Appl. Phys. A: Mater. Sci. Process. 78, 1107 (2004).

${ }^{22}$ H. Hirori, K. Matsuda, Y. Miyauchi, S. Maruyama, and Y. Kanemitsu, Phys. Rev. Lett. 97, 257401 (2006).

${ }^{23}$ D. Karaiskaj and A. Mascarenhas, Phys. Rev. B 75, 115426 (2007).

${ }^{24}$ C. Klingshirn, Semiconductor Optics, 3rd ed. (Springer, Berlin, 2007); Y. Kanemitsu, T. Nagai, T. Kushida, S. Nakamura, Y. Yamada, and T. Taguchi, Appl. Phys. Lett. 80, 267 (2002).

${ }^{25}$ R. B. Capaz, C. D. Spataru, P. Tangney, M. L. Cohen, and S. G. Louie, Phys. Rev. Lett. 94, 036801 (2005).

${ }^{26}$ J. Jiang, R. Saito, K. Sato, J. S. Park, G. G. Samsonidze, A. Jorio, G. Dresselhaus, and M. S. Dresselhaus, Phys. Rev. B 75, 035405 (2007).

${ }^{27}$ V. N. Popov and P. Lambin, Phys. Rev. B 74, 075415 (2006).

${ }^{28}$ H. Suzuura and T. Ando, Phys. Rev. B 65, 235412 (2002). 